\section{Prevención de la adicción a narcóticos dirigido a los usuarios que asisten a la consulta de traumatología de un Centro Médico}

\section{Prevention of narcotic addiction aimed at users who attend the trauma consultation of the Medical Center}

\section{Prevenção da dependência aos narcóticos destinada aos usuários que comparecem à consulta de trauma de um Centro Médico}

\author{
Elizabeth Castillo \\ leinad08_09_@hotmail.com \\ Leinad Cisneros \\ adiicisneros15@gmail.com
}

Recibido diciembre 2019 / Revisión enero 2020 / Aceptado 1 de mayo 2020
ISSN-L: $2664-3243$ pp. 95 - 103

\title{
RESUMEN
}

Introducción: Enfermería mediante el cumplimiento de su función docente promueve actitudes reflexivas en los pacientes para prevenir adicción a los narcóticos. Objetivo Información para la prevención de la adicción a narcóticos que tienen los pacientes que asisten a la unidad clínica de traumatología del Centro Médico. Materiales y métodos: Estudio descriptivo, de campo, cuya población estuvo conformada por 50 usuarios, se trabajó con la totalidad, se aplicó estadística descriptiva. Los datos se recogieron mediante cuestionario con 13 ítems con escala de Likert, la validez bajo el juicio de expertos y la confiabilidad por la prueba Alfa de Crombach. Resultados: Los resultados señalan que los pacientes ingieren analgésicos distintos a los de la prescripción médica en un $80 \%$, aumentan la dosis diaria prescrita de analgésicos narcóticos siempre en un $50 \%$ de la población. Así como también $80 \%$ manifestaron la importancia de que los profesionales de enfermería aporten a los usuarios de traumatología información sobre la prevención de la adicción a analgésicos narcóticos. Conclusión: Los pacientes cumplen más de la dosis de analgésicos indicada por los médicos, además de combinarlos con otros no prescritos y no lo reconocen como una adicción por lo que es importante la información que los profesionales de enfermería puedan aportar al respecto.

Palabras clave: narcóticos; prevención; adicción, enfermería

\begin{abstract}
Introduction: Nursing by fulfilling its teaching role promotes reflexive attitudes in patients to prevent addiction to narcotics. Objective Information for the prevention of narcotic addiction that have the patients who attend the trauma unit of the Medical Center. Materials and methods: Descriptive, field study, whose population was made up of 50 users, we worked with all, descriptive statistics were applied. The data were collected by means of a questionnaire with 13 items with a Likert scale, validity under the judgment of experts and reliability by the Alpha test of Cronbach. Results: The results indicate that patients ingest pain relievers other than those prescribed by a doctor by $80 \%$, increase the prescribed daily dose of narcotic pain relievers always by $50 \%$ of the population. As well as $80 \%$ stated the importance that nursing professionals provide trauma users with information on the prevention of addiction to narcotic pain relievers. Conclusions: The patients fulfill more than the dose of analgesics indicated by the doctors, in addition to combining them with other non-prescribed ones and do not recognize it as an addiction, so it is important the information that nursing professionals can provide in this regard.
\end{abstract}

Key words: narcotics; prevention; addiction, nursing

EC: Universidad Nacional Experimental de los Llanos Centrales "Rómulo Gallegos", Venezuela.

LC: Universidad Nacional Experimental de los Llanos Centrales "Rómulo Gallegos", Venezuela. 
EC: Universidad Nacional Experimental de los Llanos Centrales "Rómulo Gallegos", Venezuela.

LC: Universidad Nacional Experimental de los Llanos Centrales "Rómulo Gallegos", Venezuela.

\section{RESUMO}

Introdução: A enfermagem, cumprindo seu papel educacional, promove atitudes reflexivas nos pacientes para evitar o vício em narcóticos. Objetivos: facilitar informações objetivas para a prevenção de dependência de narcóticos realizadas por pacientes atendidos no centro de trauma do Centro Médico. Materiais e métodos: Estudo de campo descritivo, cuja população era composta por 50 pacientes, trabalhamos com todos, aplicamos estatística descritiva. Os dados foram coletados por meio de um questionário com 13 itens, tipo escala Likert, previamente avaliado por especialistas da área e com confiabilidade pelo teste Alpha de Crombach. Resultados: Os resultados indicam que os pacientes ingerem analgésicos diferentes dos prescritos por médicos em $80 \%$, aumentam a dose diária prescrita de analgésicos narcóticos sempre em $50 \%$ da população. Da mesma forma, $80 \%$ afirmaram a importância de que os profissionais de enfermagem fornecerem aos pacientes de trauma, informações sobre a prevenção do vício em analgésicos narcóticos. Conclusão: Os pacientes cumprem mais do que a dose de analgésicos indicada pelos médicos, além de combiná-los com outros não prescritos e não o reconhecem como um vício, por isso é importante a informação que os profissionais de enfermagem possam fornecer a esse respeito.

Palabras clave: Narcóticos; prevenção; dependência; enfermagem

\section{INTRODUCCIÓN}

$\mathrm{E}$ l dolor es un síntoma molesto que es referid por el enfermo como una molestia de intensidad variable restringida en una parte del cuerpo. Tiene su utilidad como signo de alarma de un funcionamiento anormal del organismo y como orientación de la localización de la enfermedad.

El dolor es uno de los síntomas que más sufrimiento produce en cualquier patología y constituye un problema básico de salud en todo el mundo (1), es definido como una experiencia sensorial o emocional desagradable asociada a un daño tisular real o potencial de los tejidos, u ocasionada por dicha lesión (2).

El tratamiento del dolor, ha sido prioridad desde el comienzo de la medicina y su tratamiento ha evolucionado a la par de ella. El hombre del Neolítico hace más de 9.000 años atacaba el dolor desde el aspecto físico, con plantas, sangre de animales, así como frío y calor (3). Un grupo de medicamentos utilizados para atender el dolor intenso, si no se alivia con otros, son los narcóticos o analgésicos opioides. La palabra narcótico proviene del griego narkos y significa adormecimiento o torpeza. Aunque se utilizó para denominar a los opiáceos, está más extendido su empleo en el ámbito policial y jurídico para denominar a las drogas de abuso, por lo que no parece adecuado utilizarla (4).

Los analgésicos narcóticos se pueden definir como sustancia química que induce estupor, coma o insensibilidad al dolor (5). Existen en el organismo opioides de tipo endógenos y los de tipo exógenos, ambos se unen de forma específica a los receptores opioides que, se encuentran en el cerebro, especialmente en áreas como la materia gris periacueductal y a lo largo de la médula espinal $\mathrm{y}$, en la periferia, por ejemplo, en los plexos mientéricos digestivos y articulaciones (6).

El término opiáceo, se aplica a aquellas substancias derivadas del opio, y en este grupo se encuentra la morfina, la codeína y una gran variedad de congéneres semisintéticos y sintéticos derivados de ellas, se aplica a todos los agonistas y antagonistas con actividad del tipo de la 
morfina, lo mismo que a los péptidos opioides naturales y sintéticos. También se le asocia a la endorfina que es un término genérico que se refiere a las tres familias de péptidos opioides endógenos: encefalinas, endorfinas y dinorfinas (7).

Los opioides actúan adhiriéndose a proteínas específicas llamadas receptores de opioides, que se encuentran en el cerebro, la médula espinal, el tracto gastrointestinal y otros órganos en el cuerpo. Cuando estas drogas se adhieren a sus receptores, reducen la percepción del dolor.

Los analgésicos opioides endógenos (encefalinas, endorfinas y dinorfinas), y los analgésicos exógenos naturales (morfina y codeína), así como los analgésicos opioides semisintéticos (buprenorfina) y sintéticos (fentanilo, sufentanilo, alfentanilo y remifentanilo) tienen mecanismos de acción que muestran su participación a nivel presináptico y postsináptico. A nivel presináptico inhiben la liberación de la substancia $\mathrm{P}$, también inhiben la liberación de dopamina, noradrenalina y acetilcolina en el SNC. A nivel postsináptico disminuyen la actividad de la adenilciclasa, inhiben el disparo eléctrico espontáneo inducido por la estimulación nerviosa nociceptiva y por la inyección de glutamato, reducen la velocidad de la descarga neuronal e inhiben la despolarización postsináptica (8).

El grupo de los narcóticos mencionados comprende gran variedad de drogas con efectos psicoactivos, aunque terapéuticamente no se usan para promover cambios en el humor, como los psicotrópicos, sino por otras propiedades farmacológicas: analgesia, anestesia, efectos antitusivos, antidiarreicos

Los narcóticos generan beneficios en cuanto al tratamiento eficaz del dolor, su uso, causa adicción y dependencia; perjudican en gran medida la salud de las personas, se ha señalado que las sustancias legales, como el fentanilo y los analgésicos de prescripción, están incitando el abuso y las sobredosis de opioides, lo cual puede causar alteraciones de salud, como, por ejemplo: respiratorias, frecuencia cardíaca irregular, temperatura corporal alta $\mathrm{y}$ convulsiones (9).

Los riesgos de adicción a los medicamentos de prescripción aumentan cuando se utilizan de manera distinta a las indicadas por el médico (por ejemplo, en dosis más altas, usando una vía de administración diferente, o en combinación con el alcohol u otras drogas) y al parecer los narcóticos, son las medicinas más proclives a ser empleadas de esta forma; casi 1 de cada 5 personas de Estados Unidos abusa de los medicamentos recetados, en especial los analgésicos, son el segundo tipo de droga de la que se abusa más comúnmente después de la marihuana (10).

La nación venezolana no escapa de este hecho, bien sea por el abuso de narcóticos recetados o por automedicación. Es muy común en Venezuela que al momento de estar entre amigos o familiares se hable sobre algunas afecciones y es típico que las personas acostumbren a recetar y realizar recomendaciones basados en sus propias experiencias con los analgésicos $\mathrm{u}$ otros medicamentos sin embargo, esta práctica común genera el uso y abuso de fármacos sin indicación médica.

Enfermería mediante una participación educativa, oportuna y eficaz, puede promover actitudes reflexivas en los pacientes para estar orientados a prevenir una adicción con los narcóticos, de una manera segura, esto implica la realización de varias actividades $o$ acciones importantes para satisfacer las necesidades informativas de los pacientes, en la prevención de riesgos que pudieran 
afectar su salud y estilo de vida, por la utilización indiscriminada deseos fármacos, sobre todo los de tipo oral.

Se considera prioritario que el profesional de enfermería eduque a los usuarios que deben consumir narcóticos, para así prevenir la adicción; debido a que se considera que la educación y la prevención son dos elementos centrales en toda iniciativa que tenga por objetivo desarrollar programas concretos $\mathrm{y}$ efectivos en la lucha contra la dependencia que genera la droga, sea esta lícita o ilícita.

En este sentido el personal de enfermería mediante la ejecución del rol educativo puede participar en prevención de la adicción a narcóticos dirigido a los usuarios que asisten a la unidad clínica de traumatología del Centro Médico.

\section{MATERIALES Y MÉTODOS}

$\mathrm{S}$ e realizó una investigación descriptiva, con un diseño de campo, transversal y prospectivo. La población estuvo representada por todos los usuarios internos y externos atendidos en la Unidad Clínica de Traumatología del Centro Médico, ubicado en la parroquia Guatire del municipio Zamora, estado bolivariano de Miranda, durante los meses de julio, agosto y septiembre del año 2018, según cifras aportadas por el Departamento de Estadísticas e Historias
Médicas fueron un total de doscientos cincuenta usuarios. La muestra estuvo constituida por todos los pacientes que acudieron a consulta de traumatología los tres días de la aplicación del instrumento, los cuales, fueron un total de cincuenta (50) personas. Resultando importante destacar que la selección de los sujetos, se realizó mediante el muestreo no intencionado, como criterio de exclusión se consideró solo a los pacientes menores de edad.

\section{RESULTADOS Y DISCUSIÓN}

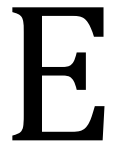
n este apartado se muestran los resultados más relevantes que fueron arrojados durante el abordaje de este estudio relacionado con la prevención de la adicción a narcóticos dirigido a los usuarios que asisten a la consulta de traumatología de un Centro Médico mediante análisis de cada gráfica mostradas.

Los datos obtenidos en relación al consumo de analgésicos distintos a los de la prescripción médica por parte de los encuestados fueron: $80 \%$ (40 sujetos) si y $20 \%$ (10 sujetos) no. Los pacientes por lo general realizan una mezcla de consumo de varios analgésicos para realizar una sinergia del medicamento indicado y la sensación de disminución del dolor. 


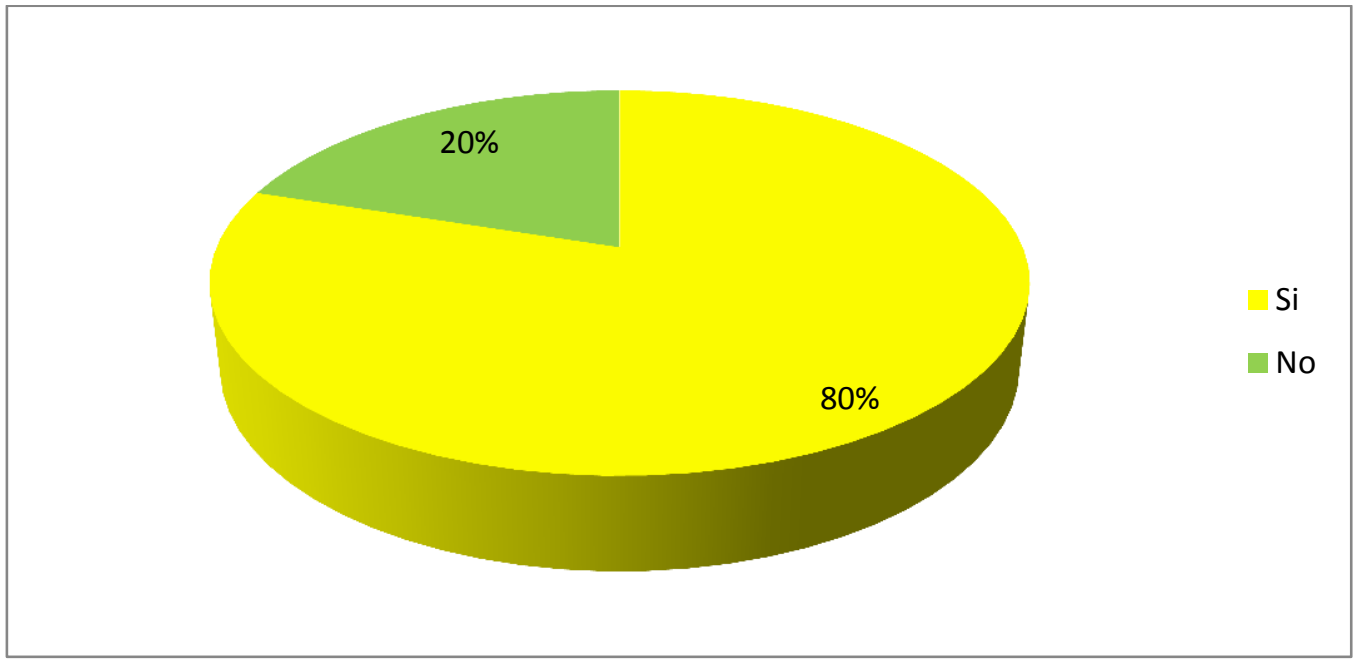

Gráfico 1. Consumo de analgésicos distintos a los de la prescripción médica. Fuente: Instrumento Aplicado

Los encuestados en cuanto al aumento de la dosis diaria de analgésicos narcóticos, opinaron; siempre 50\% (25 sujetos), algunas veces $30 \%$ (15 sujetos), $20 \%$ (10 sujetos) nunca (Gráfico 2). Por tanto, se puede referir que el $80 \%$ de los sujetos del estudio, están expuestos a la adicción a los analgésicos narcóticos debido a que están acostumbrando al organismo a recibir dosis cada vez mayores tanto en miligramos como en frecuencia de consumo. La persona acostumbra al organismo a recibir cada vez mayores cantidades de la sustancia (analgésicos), para obtener el efecto deseado, que resulta placentero, lo que se denomina tolerancia (10).

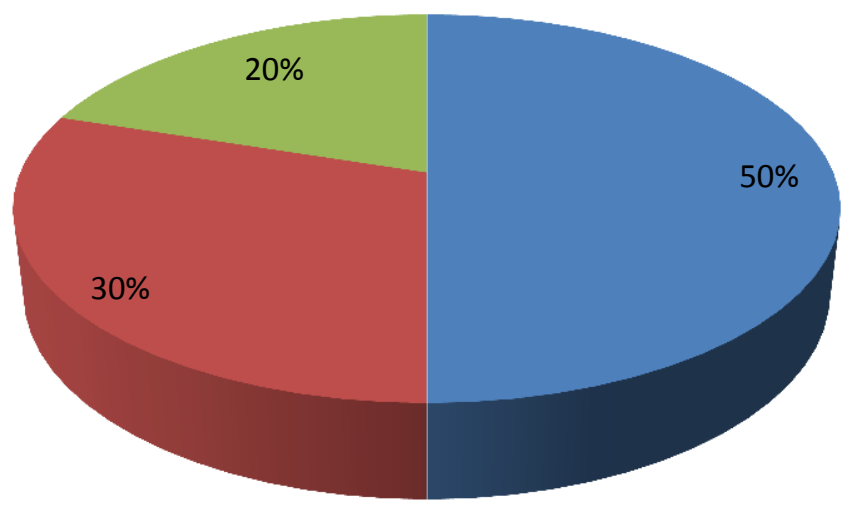

- Siempre

algunas V.

Nunca

Gráfico 2. Aumento de la dosis diaria de analgésicos narcóticos prescrita por el médico. Fuente: Instrumento Aplicado

En relación a que la adicción a los medicamentos narcóticos es una enfermedad crónica del cerebro que hace que una persona compulsivamente busque tomar mayores dosis de estos medicamentos para obtener una sensación de analgesia asociada al placer, los sujetos de la muestra opinaron: 10\% (totalmente de acuerdo, $10 \%$ de acuerdo, $20 \%$ totalmente en desacuerdo y $60 \%$ ni de acuerdo ni en desacuerdo. Opiniones que llevan a señalar que los usuarios del 
estudio tienen debilidades de conocimiento en cuanto a lo que es la adicción a los medicamentos narcóticos, lo cual puede ser un aspecto que no les permita realizar el autocuidado de la salud.

$\mathrm{Al}$ respecto, resulta importante destacar que el autocuidado es el conjunto de acciones intencionadas que realiza la persona para controlar los factores internos o externos, que pueden comprometer su vida y desarrollo posterior (9). El autocuidado, por tanto, es una conducta que realiza o debería realizar la persona para sí misma, consiste en la práctica de las actividades que las personas maduras, o que están madurando, inician y llevan a cabo en determinados períodos de tiempo, por su propia parte y con el interés de mantener un funcionamiento vivo y sano, continuar, además, con el desarrollo personal y el bienestar mediante la satisfacción de requisitos para las regulaciones funcional y del desarrollo.

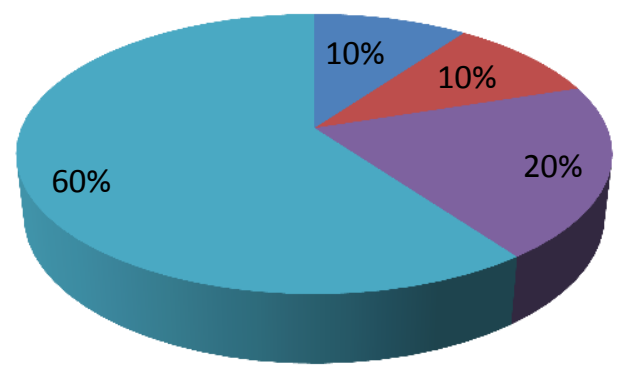

Total Acue.

De Acuerdo

En Desac.

Total Desac.

Ni Acrd.Ni D.

Gráfico 3. La adicción a los medicamentos narcóticos es una enfermedad crónica del cerebro que hace que una persona compulsivamente busque tomar mayores dosis de estos medicamentos. Fuente: Instrumento Aplicado.

Los encuestados manifestaron en relación a la importancia de que los profesionales de enfermería les aporten a los usuarios del servicio de traumatología información sobre la prevención de la adicción a analgésicos narcóticos; 80\% siempre, $20 \%$ algunas veces. Es importante que reconozcan que los profesionales de enfermería deben desempeñar el rol educativo, para prevenir las situaciones que comprometan la calidad de vida de la población e incluirlo dentro de sus actividades.

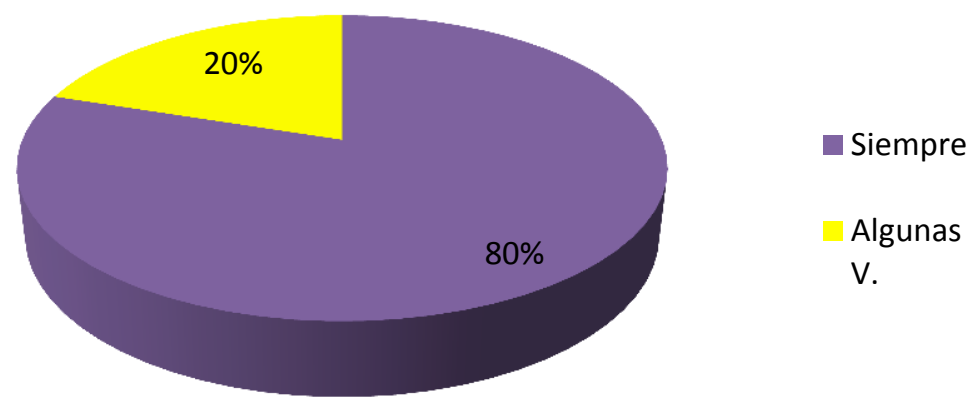

Gráfico 4. Considera importante que los Profesionales de Enfermería les aporten a los usuarios del Servicio de Traumatología información sobre la prevención de la adicción a analgésicos narcóticos. Fuente: Instrumento Aplicado 
Los enfermos encuestados se manifestaron de acuerdo a participar en el desarrollo del plan educativo dirigido a la prevención de la adicción a medicamentos narcóticos, en la distribución porcentual de los respuestas se obtuvo un si 60\% (30 sujetos), no $40 \%$ (20 sujetos). Es así como se puede señalar, que el plan antes nombrado, tiene factibilidad de realización por la participación de los consultados.

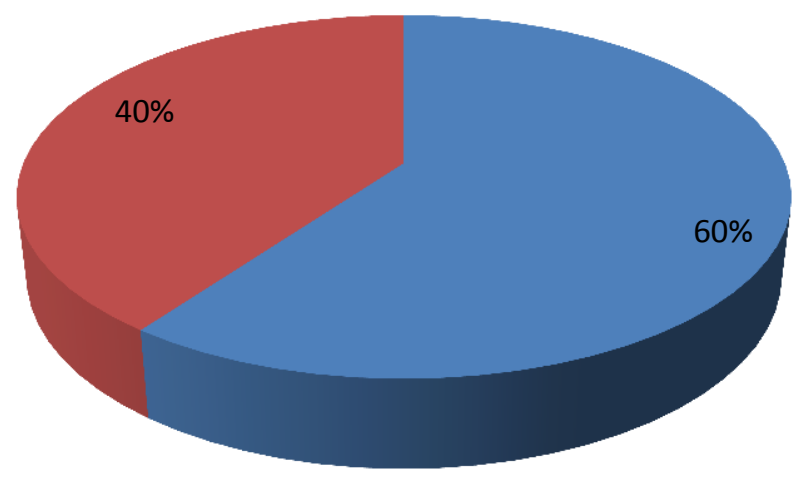

Gráfico 5. Participaría en el desarrollo del plan educativo dirigido a la prevención de la adicción a medicamentos narcóticos. Fuente: Instrumento Aplicado

\section{Discusión}

Los analgésicos opioides son seguros cuando se ingieren o administran por un período de tiempo corto y siguiendo las indicaciones del médico, pero como además de calmar el dolor generan euforia, a veces se los utiliza en forma inapropiada, es decir, se toman en forma diferente a la indicada, o en mayores dosis. Pero el consumo regular-aun cuando se sigan las instrucciones del médico-puede llevar a la dependencia, y si se los usa en forma inapropiada, los analgésicos opioides pueden llevar a situaciones de sobredosis y causar la muerte.

En relación a los factores que intervienen en la adicción a estos medicamentos, a medida que el consumo de los opiáceos aumenta, el organismo necesita cada vez mayores cantidades de la sustancia, para obtener el efecto deseado de disminución del dolor, lo que resulta placentero, esto se denomina tolerancia. Es una condición caracterizada por disminución en la capacidad de respuesta, la cual se adquiere después de un contacto repetido con determinado medicamento o con otros de similar actividad farmacológica.

La tolerancia farmacológica se caracteriza por la necesidad de aumentar las dosis sucesivas para provocar efectos o duración a los logrados al inicio (11). Es un estado de adaptación caracterizado por la disminución del efecto a la misma cantidad de droga consumida o por la necesidad de una dosis mayor para provocar el mismo grado de efecto (12).

Diversos factores intervienen en las adicciones, entre ellos se encuentran los que involucran al funcionamiento cerebral. También existen particularidades genéticas que afectan el funcionamiento del cerebro y condicionan la aparición de adicciones. Las adicciones, a su vez, alteran la funcionalidad cerebral dañando, entre otras, las funciones ejecutivas; lo que produce un círculo vicioso que perpetúa la propia adicción (13). 
Un problema común en la prescripción de analgésicos de tipo narcóticos es que la persona que consume una misma dosis de un medicamento de este tipo, paulatinamente, deja de sentir los efectos iníciales y para sentir el mismo efecto deseado, con el tiempo, tiene que incrementar la dosis del consumo de la droga (12). Intervienen en la adicción a los narcóticos: las condiciones ambientales y la exposición a estímulos posteriores al consumo, pues con el uso repetido, el adicto queda condicionado a las señales asociadas al consumo de droga.

La edad y el género también juegan un rol importante. Las mujeres tienden a ser más susceptibles al abuso de analgésicos narcóticos debido a las diferencias hormonales y su tamaño corporal en relación con los hombres. Con respecto a la edad, los ancianos tienen barreras hemato encefálicas menos eficaces, lo que se traduce en una menor resistencia a diferentes sustancias incluidos los opiáceos, por lo tanto, hay una mayor probabilidad de consumo, uso y abuso de los narcóticos por parte de esta población (13). Los resultados muestran que la mayoría de las personas modifican la prescripción médica y el uso de los analgésicos narcóticos aumentando la frecuencia, la dosis o usándolo en combinación con otros analgésicos para obtener la disminución del dolor, esto causa de manera inconsciente problema de tolerancia medicamentosa lo que conlleva a la adicción a estos fármacos.

La prevención de la dependencia a los analgésicos narcóticos, es importante en los casos de los usuarios de los centros de salud y la educación es la estrategia cónsona para tal fin

\section{CONCLUSIONES}

$\mathrm{E}$ n relación a los narcóticos que son utilizados por los usuarios que asisten al a la Unidad Clínica de Traumatología del Centro ubicado en la parroquia Guatire del Municipio Zamora, Estado Miranda, se establece que tienen información sobre el cómo debe ser el uso de los narcóticos, no obstante, esto no resulta ser suficiente para poder establecer que ellos conocen del tema y que la información que maneja pueda ser aceptable debido a las consecuencias que estos generan para la salud.

Al describir las reacciones adversas ante el consumo de narcóticos sin y con indicación médica, se detecta que la información que poseen los pacientes es deficiente en cuanto a: problemas que se pueden presentar en el organismo, personalidad, consciencia, entorno social, entre otros factores sociales.

Se hace necesario la realización de un plan educativo para la prevención de la adicción a narcóticos dirigido a los usuarios que asisten a la Unidad Clínica de Traumatología del Centro Médico, lo que permitirá que los pacientes puedan contar con la información necesaria y así evitar una adicción a estos medicamentos.

Es por esto, es relevante implementar acciones dentro de la institución para promover la realización de talleres en los que se les ofrezca información a los pacientes, así como personal docente, sobre los narcóticos, donde todos puedan aclarar sus dudas en cuanto al uso de los medicamentos.

- Conflicto de intereses. Ninguno declarado por las autoras

- Financiación. Ninguna declarada por las autoras

- Investigación realizada dentro de los estándares éticos

\section{REFERENCIAS}

1.- Martínez C. Collado F. Rodríguez J. y Moya J. El alivio del dolor: un derecho humano universal. Rev. Soc. Esp. Dolor. 2015(Consultado 02 agosto 2017); 
22(5):224-230. Disponible en: http://scielo.isciii.es/pdf/dolor/v22n5/07 _aespecial.pdf

2.- SED Sociedad Española del Dolor. Manual de Medicina del Dolor Fundamentos, evaluación y tratamiento. España: Médica Panamericana; 2016 3.- Pérez-Cajaraville J, Abejón D, Ortiz J, Pérez J. Pain and its treatment over history. Rev. Soc Esp Dolor 2005; 12:373384. (Consultado 02agosto 2017);

4.- Flórez J, Armijo JA, Mediavilla A. Fármacos analgésicos opioides. Farmacología humana. 4a ed. Barcelona: Masson; 2003; 461-478.

5.-rganización de las Naciones Unidas. Terminología e información sobre drogas. 3era Ed (consultado 20 junio 2017) Disponible

en:https://www.unodc.org/documents/sc ientific/Terminology_and_Information_on_ Drugs_S_3rd_Edition.pdf

6.- Álvarez Y, Farre M. Farmacología de los opioides. Adicciones.2005; 17(2):21. Disponible en: https://www.redalyc.org/pdf/2891/2891 22022016.pdf

7.- Villarejo-Díaz M. Murillo-Zaragoza J. Alvarado-Hernández H. Farmacología de los agonistas y antagonistas de los receptores opioides. Educación e Investigación Clínica. 2000; 1(2):106-137 8.- Navas M, Tellez A, Rojas D. Usos terapéuticos potenciales de los antagonistas opioides: Fisiopatología y evidencia preclínica. Rev. Colomb. Cienc. Quím. Farm. 2015; 44(3):322-358. Disponible en http://www.scielo.org.co/pdf/rccqf/v44n 3/v44n3a05.pdf

9.- Álamo C. ¿Existen argumentos para combinar un opioide con otro opioide? Rev. Soc. Esp. Dolor [Internet]. 2016; 23(3):121-126 [citado 2018 Abr 17]. Disponible en: http://scielo.isciii.es/scielo.php?script=sci _arttext\&pid=S1134$80462016000300001 \& \operatorname{lng}=\mathrm{es}$.

10.- Centro Nacional de Información de Ciencias Médicas. Tolerancia farmacológica. Glosario de términos farmacológicos. 2011. Consultado el 27 de enero de 2018. Disponible en: http://glosario.sld.cu/terminosfarmacologicos/2011/05/03/toleranciafarmacologica/

11.- Aguilar E. Mendoza M. Valdez G. López M. Camacho R. Disfunción cerebral Rev Esp Méd Quir 2012; 17(2):119-124

12.- Carneiro C. Ashmawi H. De Paula I. Sexo y Percepción del Dolor y Analgesia. Rev Bras Anesthesia. 2011; 61(6): 449-458 13.- Dobin DG, Andrews R, BecKer WC. Prescribing opioids in primary care: Safely starting, monitoring, and stopping. Cleveland Clinic Journal of Medicine 2016; 83(3):207-15 